$1^{\text {st }}$ ARENA Conference on Astronomy at CONCORDIA

N. Epchtein and M. Candidi (eds)

EAS Publications Series, 25 (2007) 1-4

\title{
ARENA: TOWARD A EUROPEAN ASTRONOMICAL FACILITY AT DOME C CONCORDIA
}

\author{
N. Epchtein ${ }^{1}$
}

Abstract. Opening talk of the Conference and acknowledgments.

\section{Opening Address}

I am very glad to open this 1st ARENA Conference in the pleasant environment of French Brittany, and I would like to thank warmly the CNRS Conference Center of Roscoff and Prof. B. Kloareg, head of the Station Biologique, for kindly hosting this Conference on "Large Astronomical Infrastructures at CONCORDIA, prospects and constraints for Antarctic optical/IR Astronomy".

I hope this will be a very useful and interactive meeting for our 120 attendees that I thank very much for their participation. This first Conference is not strongly focused on particular science or technical topics but is rather aimed at gathering engineers and researchers who are not used to work together. It is a major purpose of this European network to set up new collaborations and to create new links between laboratories, industrial companies, research institutes and agencies. On the other hand, the second ARENA Conference to be held in September 2007 in Potsdam will be mainly devoted to highlight the top science cases.

We have not chosen this venue at random, but essentially to be near to the IPEV headquarters in Brest and thus to stimulate interactions between astronomers and the French operator of CONCORDIA, and this will offer us the opportunity to visit this Institute before enjoying the Conference dinner at Oceanopolis.

Five years ago, Antarctic Astronomy was considered in France and in Europe as a rather exotic discipline reserved to pioneers in quest of new sensations and feelings, but few were really thinking that it could seriously become an issue in the prospect of next decade astronomy and considered for the installation of large instruments that could achieve science that would normally request a space mission.

\footnotetext{
1 Coordinator of the ARENA network, CNRS-LUAN-UNSA, Parc Valrose, 06108 Nice Cedex 2, France;

e-mail: epchtein@unice.fr
}

(C) EAS, EDP Sciences 2007 DOI: $10.1051 /$ eas:2007063 
Since that time and thanks to the strong involvement of several laboratories and of our pioneer Antarctic astronomers, the interest of astronomers for Dome $\mathrm{C}$ has considerably evolved, essentially thanks to the first very encouraging results of site testing. Useless to repeat that Dome $\mathrm{C}$ is cold and dry and thus very well suited to thermal infrared astronomy, moreover, it provides also excellent atmospheric turbulence conditions above a thin turbulent layer offering unique conditions for high angular optical/NIR observations. This turbulent layer is some 30 meters height, raising the question of how to install a telescope and stabilize it atop a tower. This will be the matter of a dedicated presentation and discussions during this conference. This is my first point.

Second point is the fact that French and Italian Polar Agencies have demonstrated their capability to deploy all the logistics and expertise necessary to run a scientific station in Polar conditions all year round, and especially during the Winter with the first winterover in 2005 and thanks to the extraordinary willingness of pioneering staff astronomers such as Abdelkrim Agabi. Claire Le Calvez, the first woman to winterover at Dome $\mathrm{C}$, will give us a taste of what a winterover at Dome C looks like.

Third important point is the fact that European astronomers who believe that the Antarctic site is absolutely outstanding have begun to realize that coordination and sharing of expertise is a prerequisite to undertake feasibility, and later on, design studies of large projects, there, rather than to work independently on individual projects. The invaluable contribution of our Australian colleagues is also very much welcome in this respect, as well as other possible partnerships outside the EU, such as US, Japan, China, etc..

I hope that the ARENA network sponsored by the European Commission has started to help in the way of collaboration, not only within Europe, but also with Australia and that this first Conference will open new opportunities and prospects to strengthen these interactions in the coming years. It is certainly too early to start evaluating its impact, but my feeling is that after raising more issues than solving them, we are progressing in the right way since many of us did not even know about each other activity in polar astronomy just a few months ago.

Most of you, as consortium members, know what ARENA is, its aims and objectives. In just a sentence, ARENA is primarily aimed to identify needs and develop actions to structure the European optical/IR astronomical community around several large projects at Dome C CONCORDIA.

This means that except for site testing instrumentation, we are not supposed to consider nor support small experiments, but rather to arouse a consensual interest for one or two world-class projects and to define their road map of development during the coming decade from the point of view of their scientific impact, of the technical challenge that they will implicate and of the supplementary logistics effort that they will require.

Before dreaming of a kilometric interferometric array or of an ELT on-ice, there are however many intricate questions that must be addressed and hopefully thoroughly investigated. This first Conference is mostly aimed at reviewing a preliminary list of questions about the feasibility of such projects (a roadmap) and 
to start the reflection on how far we can realistically go in such harsh conditions to do world-class astronomy (the science case) within the present and future context of space missions such as JWST, Darwin, or large ground based projects such as the ELTs.

We will definitely not answer all these questions during this conference, of course, and we will have 2 more years to go much deeper into several specific issues such as consolidating a strong science case, robotization, or building of towers on ice, as we started to do this year with 2 formerly held ARENA workshops dedicated to interferometry and wide field astronomy.

Therefore, I strongly suggest attendants and partners to propose new meetings of various sizes in the future.

This 1st conference is deliberately multidisciplinary and will involve

- site qualification status,

- science cases,

- instrumental developments.

Putting so many topics in a 3 and half day meeting was really quite challenging, but I have the feeling that at some point it is important that we open our minds to the real constraints that exists at Dome $\mathrm{C}$, this will hopefully temper dreams of presently unrealistic endeavours, to evaluate the limits of what can really be done in the present conditions and whether or not, from the astrophysical point of view, it is worth developing pathfinders, demonstrators or other intermediate size instruments that would not only cost significant amount of money, but also employ and lock expert manpower on not totally scientifically convincing projects. At a certain level of funding, demonstrators or pathfinders should also bring real astrophysical breakthroughs. Are we convinced that they will do?

I will briefly comment on the programme of this Conference, now.

This first day will be essentially dedicated to present the current status of the site qualification and to propose further developments in that respect, and notably how to give a fluent access to this essential information within the consortium and the community. These data are obviously of common interest and are a prerequisite to further design studies of instruments.

The second day will be mainly dedicated to review the most compelling science that could be achieved in antarctic conditions from the search for exoplanets to cosmology.

The last 2 days are dedicated to the concepts of possible instruments up to the possibility of studying an ELT on ice, for which there are quite a lot of strong arguments, although far from matching the capacity of the present infrastructure and logistics at Dome C.

Finally, I'd like to stress the fact that we have also to start thinking of what will happen after ARENA in the context of the forthcoming European Framework Program (FP7). I would like to express my wish to start discussing about a more ambitious EC proposal such as an I3 that would broaden our scope to other topics 
among which, obviously, submillimeter and millimeter astronomy and atmospheric sciences, involving not only networking of partners, but also joint research activities of RTD as well as transnational access. We have a little bit more than one year to prepare such a proposal.

It is a great pleasure for me to thank the whole staff of the Conference Center of CNRS in Roscoff for the fine practical organization of this meeting, and for the burden of works it has caused to them during the last weeks of preparation, more specifically, Ms. N. Sanséau and B. Guézennec.

I would like to thank Dr. Gérard Jugie, Director of IPEV and all his staff for organizing the visit of their Institute in Brest and the staff of Oceanopolis in Brest for allowing us a special visit of this amazing discovery Park and organizing the Conference dinner.

The staff of the Laboratoire Universitaire d'Astrophysique de Nice is warmly thanked for their help in preparing the local and remote organization, and more specifically, Ms. M. AdrianScotto, D. Sanchèz-Sierra, Marie-Claude Pophillat, Valérie Chéron, and a special mention to our Project Manager, Ms. Marie-Laure Péronne for her tireless dedication to the preparation and the success of this conference.

I thank the Scientific Organizing Committee for producing a well balanced and highly exciting program, all the speakers and those who kindly accepted our invitation; many thanks also to Mark McCaughrean for preparing the summary of the Conference with strong, clever and timely statements.

Finally, we are indebted to the European Commission for sponsoring this Conference and fully supporting the cost of its organization under contract RICA 2006-026150. We specially thank Dr. Elena Righi-Steele, Project Officer, for helping us in the management of this network. 